This document is published in:

García-Pedrajas, N. et al. (eds.) (2010) Trends in Applied Intelligent Systems: 23rd International Conference on Industrial Engineering and Other Applications of Applied Intelligent Systems, IEA/AIE 2010, Cordoba, Spain, June 1-4, 2010, Proceedings, Part III. (Lecture Notes in Computer Science, 6098) Springer, pp. 470- 477.

DOI: http://dx.doi.org/10.1007/978-3-642-13033-5_48

(C) 2010 Springer-Verlag Berlin Heidelberg 


\title{
Finding an Evolutionarily Stable Strategy in Agent Reputation and Trust (ART) 2007 Competition
}

\author{
Javier Carbo and José Manuel Molina \\ Group of Applied Artificial Intelligence, Computer Science Department, University \\ Carlos III of Madrid \\ \{javier.carbo, josemanuel.molina\}@uc3m.es
}

\begin{abstract}
Our proposal is to apply a Game Theoretic approach to the games played in Agent Reputation and Trust Final Competitions. Using such testbed, three international competitions were successfully carried out jointly with the last AAMAS international Conferences. The corresponding way to define the winner of such competitions was to run a game with all the participants (16). Our point is that such game does not represent a complete way to determine the best trust/reputation strategy, since it is not proved that such strategy is evolutionarily stable. Specifically we prove that when the strategy of the winner of the two first international competitions (2006 and 2007) becomes dominant, it is defeated by other participant trust strategies. Then we found out (through a repeated game definition) the right equilibrium of trust strategies that is evolutionarily stable. This kind of repeated game has to be taken into account in the evaluation of trust strategies, and this conclusion would improve the way trust strategies have to be compared.
\end{abstract}

\section{Introduction}

The way agents achieve cooperation solving complex tasks is a design key factor in MultiAgent Systems. However, since Agent Systems intend to be open, agents have to establish some kind of social control that could be defined as designed or emergent [1]. The former implemented through Electronic Institutions, such as Certification Authorities, when trust/ is concluded from the observation of universal and objective norms, but in many real-world interactions trust is emergent, depends on local and subjective evaluations shared between partners (reputation). In recent years, trust/reputation research community has grown a lot, many trust/reputation models have been proposed [5]. Since it was very difficult to compare their respective performances as many ad-hoc implementations and metrics have been applied, a testbed platform for agent trust/reputation models was developed: the Agent Reputation and Trust (ART) Testbed [2] ${ }^{1}$. Using such testbed, three international competitions were successfully carried out jointly with the last AAMAS international Conferences. During these years the ART

\footnotetext{
${ }^{1}$ http : //megatron.iiia.csic.es/art - testbed
} 
testbed has been used by dozens of researchers, and the ART-testbed members have discussed, patched and updated the platform using the feedback from the Competitions (see discussion notes on ART web page) and from the agent trust community (through the discussion Board of ART). These criticism produced some changes in protocols [6], and outlined new directions of work [4]. This criticism is essentially focused on the scalability of ART games since with more agents the right use of reputation would make more sense playing an increasing role to win the game. Additionally a differentiation between the way trust is acquired/modeled/updated (trust model) and the way trust is used/applied in decisions (trust strategy) would be desirable.

This paper suggests a new way ART can be used in competitions to evaluate agents. In section 2 we explain our domain: the ART testbed. Afterwards, in section 3, We show how a game-theoretic approach can be applied in this domain. In [6] and [4] did not even mention the possibility of applying a game-theoretic approach, they just remark the needing of thinking on new different ways to evaluate trust/reputation models. Our contribution is to define a way to determine the ability of a trust strategy to win when it is dominant in the society of agents. With such intention, we propose in section 4 a new different kind of repeated games with ART testbed. In order to achieve this goal the corresponding games have been run with the participant agents of ART competitions in section 5 .

\section{ART Testbed}

The ART testbed compared different trust strategies using reputation models in the art appraisal domain. In this domain, agents are players/competitors that appraise paintings and implement trust strategies. Figure 1 shows an outline of ART domain.

In each timestep, the simulator engine presents each appraiser agent with paintings (generated by the simulation engine) to be appraised, paying a fixed fee $\mathrm{f}$ for each appraisal request. Very close valuations of paintings to the real value would lead to more future clients, and therefore to more earnings to win the competition. The corresponding steps of a turn in ART games is shown in figure 2 .

Each painting belongs to an era among a set of artistic eras while agents have different levels of expertise (ability to appraise) in each era. An agent can appraise its own paintings and may request opinions (at a fixed cost) from other appraisers to get its valuation of the painting close to the real value (specially useful in the eras where the agent has low expertise). An agent can act also as provider of appraisals in response to opinion (about paintings) requests from other agents Additionally, an agent can similarly request reputation information about other appraisers (at a fixed and much lower cost than opinions). The winner of an ART game is the agent who earned more money along the number of iterations that were run in the game. Such earnings come from different sources: paintings appraised to the own clients (Client Fee), paintings appraised to other appraiser agents (Opinion Cost) and reputations shared with other appraiser 


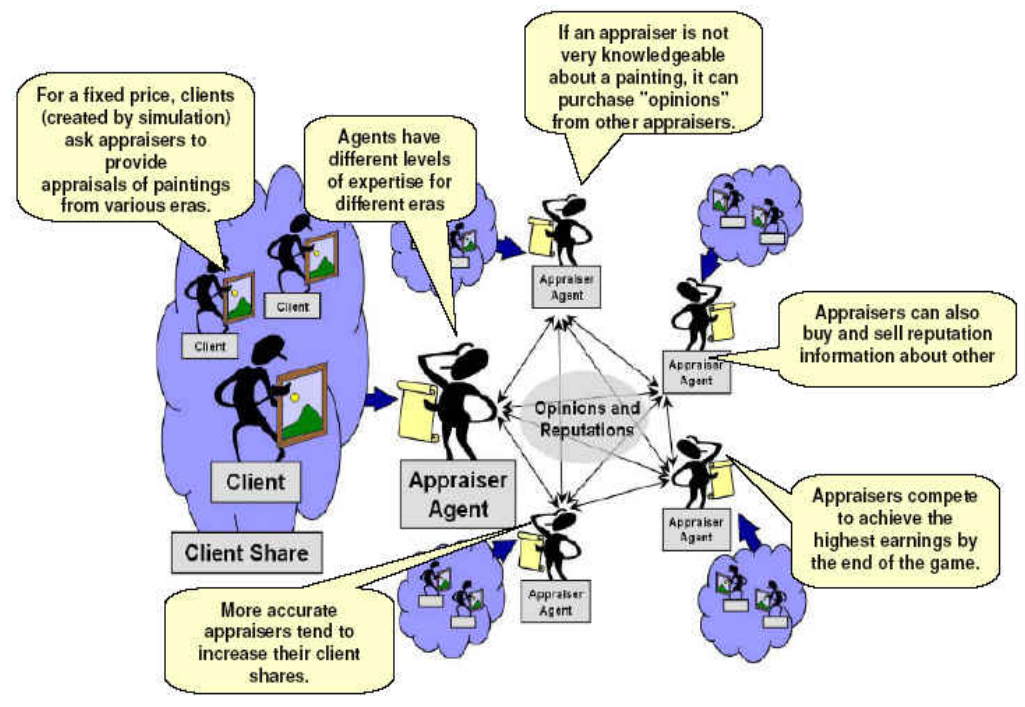

Fig. 1. ART domain outline. Source [2]

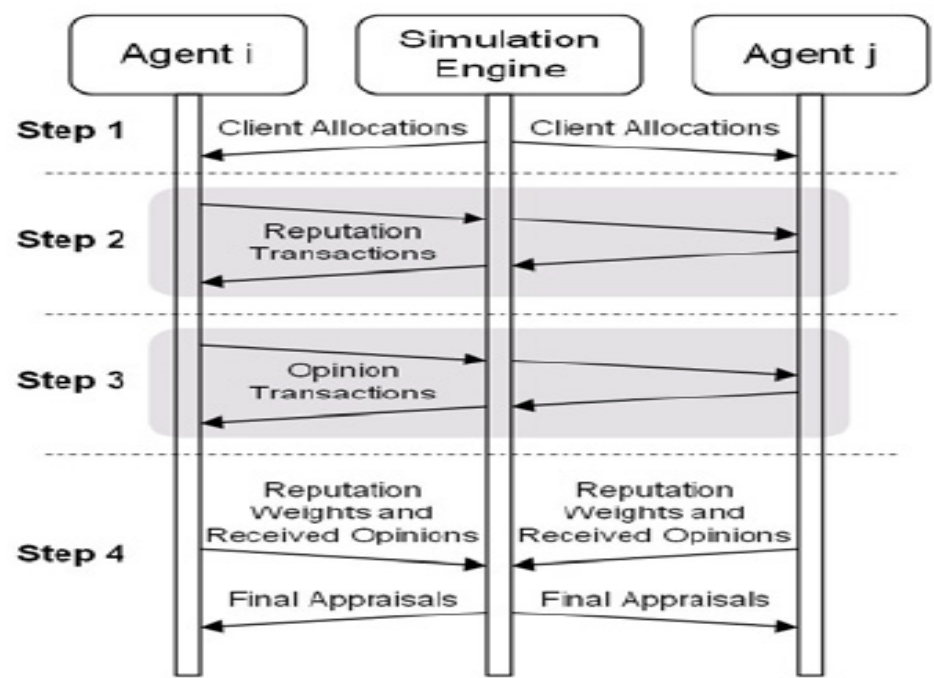

Fig. 2. Steps of a gameturn in ART domain. Source: [3] 
agents (Reputation Cost), where Client Fees are the main source of income since: Client Fee $>>$ Opinion Cost $>>$ Reputation Cost.

\section{Evolutionarily Stable Trust Strategies in ART}

\subsection{Trust and ESS}

In game theory and behavioural ecology, an evolutionarily stable strategy (ESS) is a strategy which, if adopted by a population of players, cannot be invaded by any alternative strategy. An ESS is a Nash equilibrium which is "evolutionarily" stable meaning that once it is fixed in a population, natural selection alone is sufficient to prevent alternative (mutant) strategies from successfully invading. The ESS was developed in order to define a class of solutions to game theoretic problems, equivalent to the Nash equilibrium, but which could be applied to the evolution of social behaviour in animals. Our proposal is to apply this Game Theoretic approach to the games played in Agent Reputation and Trust Final Competitions. Although in ART games there is no reproduction neither evolution, this approach make sense since the goal of trust strategies is to establish some kind of social control over malicious/distrustful agents through the exchange of local and subjective evaluations between partners (the so called reputation in ART terms). So the idea of applying trust strategies in agent societies is to filter out the agents who do not behave properly in such society. Therefore, we could state that the loser of the direct confrontation between/among trust strategies would be expelled out of such society of agents. We can even think in terms of evolution if we assume that agents may change of trust strategy. Following that line, it seems to be reasonable that agents with a failing trust strategy would get rid of it and they would adopt a successful trust strategy in the future. Through this analogy we can define a repeated game in ART domain that would allow us to evaluate the ability of participant agents in past competitions to be an evolutionarily stable trust strategy. Furthermore, we can define as an evolutionarily stable trust strategy is a strategy which, if becomes dominant (adopted by a majority of agents) can not be defeated by any alternative strategy.

\subsection{Repeated Game Definition to Find a ESS in ART}

Using ART testbed, three international competitions were successfully carried out jointly with the last AAMAS international Conferences. The corresponding way to define the winner of 2007 competitions was to run the same game with all the participants (16) several times (until 10 times) plus some dummy agents (9) with enough timesteps per game (defined as 60 minuted length to complete each game $)^{2}$. Our point is that such game does not represent a complete way to determine the best trust strategy, since it is not proved that such trust strategy is evolutionarily stable. Therefore we propose a simulation of the evolution of

${ }^{2}$ http://megatron.iiia.csic.es/art - testbed/competition2007.html 


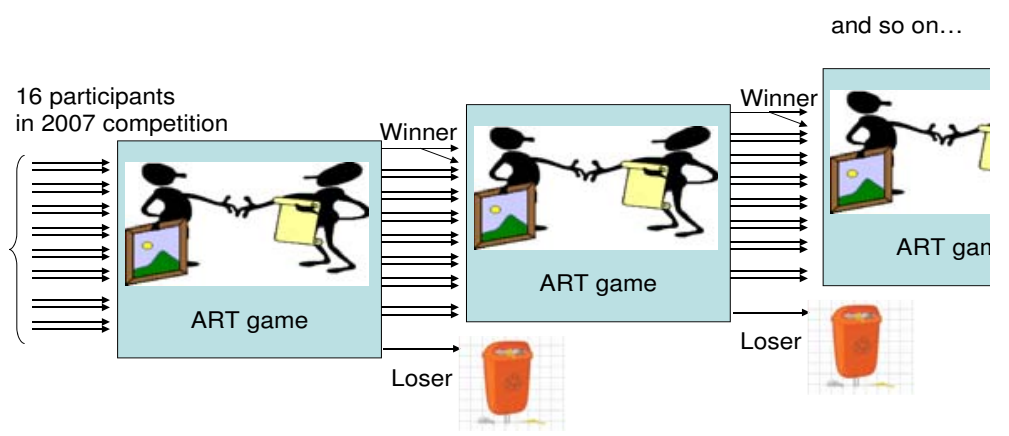

Fig. 3. Evolution Simulation in ART games

a society of ART participant agents through a set of consecutive games, where evolution is implemented assuming that agents with a failing trust strategy would get rid of it and they would adopt a successful trust strategy in the next ART game. Where we consider a failing trust strategy the one who lost (earning less money than the others) the last ART game, and we consider the successful trust strategy to the one who won the last ART game (earning more money than the others). By this way replacing in consecutive games the participant who lost the game by the one who won it. We will then find out if there is a trust strategy among the participant agents in ART competitions that is evolutionarily stable. An outline of the ART game defined to simulate a evolutive society of agents applying trust strategies is shown in figure 3 .

\subsection{Finding a ESS among 2007 ART Competitors}

Once the type of game to be run has been defined, we have applied it to the participant agents of 2007 ART competition (the code of last competition (2008) participants is not public and we want our experiments to be repeatable). First we show in table 1 the participants and the resulting earnings of such competition $^{3}$ :

As we consider these participants (without dummy agents) as the first game in our evolutive simulation, then, if the relative positions are similar we have 2 iam2 agents, no xerxes agent and the other 14 agents as participants of the second game. We proceed in same way including an extra winner agent and excluding the loser agent in consecutive games. Next we show in table 2 the agents that win and lose each consecutive game with the corresponding earnings. The earnings showed in table 2 are computed in same way as the competition, as a normalized bank balance (bank balance divided by number of timesteps in the game), and game length and game repetition defined in the same way $(60 \mathrm{~m}$ maximum, 10 times) so all the experiments shown here can be easily repeated. As it was expected, since there are no dummies to easily cheat, the earnings of

${ }^{3}$ http://megatron.iiia.csic.es/art - testbed/competition_results2007.html 
Table 1. 2007 ART Competition Results

\begin{tabular}{|c|c|c|c|}
\hline Rank & Agent name & Affiliation & Earnings \\
\hline 1 & iam2 & Univ. Southampton & 539377 \\
\hline 2 & jam & Univ. Tulsa & 353700 \\
\hline 3 & blizzard & Bogazici Univ. & 335933 \\
\hline 4 & zecariocales & Pontificia Univ. Rio & 319564 \\
\hline 5 & spartan & Univ. Girona & 311777 \\
\hline 6 & artgente & Univ. Trento & 298897 \\
\hline 7 & uno & Univ. Girona & 293324 \\
\hline 8 & reneil & Nanyang Tech. Univ. & 269905 \\
\hline 9 & marmota & Univ. Girona & 264356 \\
\hline 10 & novel & Univ. Murcia & 229501 \\
\hline 11 & alatriste & Univ. Carlos III Madrid & 225276 \\
\hline 12 & rex & Univ. Warwick & 211467 \\
\hline 13 & IMM & Univ. Carlos III Madrid & 200440 \\
\hline 14 & lesmes & Univ. Carlos III Madrid & 183655 \\
\hline 15 & agentevicente & Univ. Carlos III Madrid & 181932 \\
\hline 16 & xerxes & U.S. Airforce Research Lab. & 148610 \\
\hline
\end{tabular}

Table 2. Evolution Simulation Results

\begin{tabular}{|c|c|c|c|c|}
\hline Game Number & Winner & Earnings & Loser & Earnings \\
\hline 1 & iam2 & 17377 & xerxes & -8610 \\
\hline 2 & iam2 & 14321 & lesmes & -13700 \\
\hline 3 & iam2 & 10360 & reneil & -14757 \\
\hline 4 & iam2 & 10447 & blizzard & -7093 \\
\hline 5 & agentevicente & 8975 & Rex & -5495 \\
\hline 6 & iam2 & 8512 & alatriste & -999 \\
\hline 7 & artgente & 8994 & agentevicente & 2011 \\
\hline 8 & artgente & 10611 & agentevicente & 1322 \\
\hline 9 & artgente & 8932 & novel & 424 \\
\hline 10 & iam2 & 9017 & IMM & 1392 \\
\hline 11 & artgente & 7715 & marmota & 1445 \\
\hline 12 & artgente & 8722 & spartan & 2083 \\
\hline 13 & artgente & 8966 & zecariocales & 1324 \\
\hline 14 & artgente 8372 & 7285 & iam2 & 2599 \\
\hline 15 & artgente & 7475 & iam2 & 2298 \\
\hline 16 & artgente & 8384 & UNO & 2719 \\
\hline 17 & artgente & 7639 & iam2 & 2878 \\
\hline 18 & iam2 & 6279 & JAM & 3486 \\
\hline 19 & iam2 & 14674 & artgente & 2811 \\
\hline 20 & artgente & 8035 & iam2 & 3395 \\
\hline
\end{tabular}


Table 3. Comparison of Rankings of agents

\begin{tabular}{|c|c|c|c|}
\hline Competition Rank & Evolution Rank & Agent name & Excluded in game number \\
\hline 6 & 1 & artgente & - \\
\hline 1 & 2 & iam2 & - \\
\hline 2 & 3 & JAM & 18 \\
\hline 7 & 4 & UNO & 16 \\
\hline 4 & 5 & zecariocales & 13 \\
\hline 5 & 6 & spartan & 12 \\
\hline 9 & 7 & marmota & 11 \\
\hline 13 & 8 & IMM & 10 \\
\hline 10 & 9 & novel & 9 \\
\hline 15 & 10 & agentevicente & 8 \\
\hline 11 & 11 & alatriste & 6 \\
\hline 12 & 12 & rex & 5 \\
\hline 3 & 13 & Blizzard & 3 \\
\hline 8 & 14 & reneil & 2 \\
\hline 14 & 15 & lesmes & 1 \\
\hline 16 & 16 & xerxes & \\
\hline & & & \\
\hline
\end{tabular}

agents are much lower than those of the competition and the differences between winners and losers becomes closer in the last games.

Specifically with this experiment we have proved that although the strategy of the winner of the 2007 international competitions spreads in the society of agents (until 6 agents implementing iam2 trust strategy out of 16 participant agents), it never becomes dominant (there is no majority of iam2 agents). In fact it is defeated by other trust strategy artgente, which becomes dominant (11 artgente agents out of 16). Therefore iam2 is not an evolutionarily stable trust strategy, so its superiority as winner of 2007 competition is, at least, relative. We also found out that the right equilibrium of trust strategies that form an evolutionarily stable society is composed by 10-11 Artgente agents and 6-5 iam2 agents. Finally, from the order in which agents are excluded from the society, we can propose an alternative ranking of trust strategies in table 3 which :is very different from the competition ranking.

\section{Conclusions}

Due to the relative success of the trust and reputation research, and specifically of ART testbed, a good design foundation of fair comparisons among trust strategies will spread the inclusion of reputation and trust communications into more general service-oriented systems that would be truly distributed. According to that intention, we have defined what an evolutionarily stable strategy would be in trust domain, and how it can be proved in ART competitions through a repeated game with a simulation of evolution. We have applied such game definition to the participant agents of 2007 competition and we found out that 
the winner of such competition was not implementing an evolutionarily stable strategy. As a conclusion we stated that this kind of repeated game has to be taken into account in the evaluation of trust strategies, and this conclusion would improve the way trust strategies have to be compared, not just thinking about the testbed, also about the games definition.

\section{Acknowledgments}

This work was supported in part by Projects CICYT TIN2008-06742-C02-02/ TSI, CICYT TEC2008-06732-C02-02/TEC, SINPROB, CAM MADRINET S-0505/TIC/0255 and DPS2008-07029-C02-02.

\section{References}

1. Conte, R., Paolucci, M.: Reputation in Artificial Societies. Kluwer Academic Publishers, Dordrecht (2002)

2. Fullam, K., Klos, T., Muller, G., Sabater, J., Schlosser, A., Topol, Z., Barber, K.S., Rosenschein, J., Vercouter, L., Voss, M.: A specification of the agent reputation and trust (art) testbed: Experimentation and competition for trust in agent societies. In: The Fourth International Joint Conference on Autonomous Agents and Multiagent Systems (AAMAS 2005), pp. 512-518 (2005)

3. Fullam, K., Klos, T., Muller, G., Sabater, J., Topol, Z., Barber, K.S., Rosenschein, J., Vercouter, L.: The agent reputation and trust (art) testbed architecture. In: Workshop on Trust in Agent Societies at The Fourth International Joint Conference on Autonomous Agents and Multiagent Systems (AAMAS 2005), pp. 50-62 (2005)

4. Gomez, M., Sabater-Mir, J., Carbo, J., Muller, G.: Improving the art testbed, thoughts and reflections. In: Procs. of 12th CAEPIA Conference, pp. 1-15 (2007)

5. Ramchurn, S.D., Huynh, D., Jennings, N.R.: Trust in multi-agent systems. Knowl. Eng. Rev. 19(1), 1-25 (2004)

6. Sabater, J., Gomez, M., Muller, G., Carbo, J.: Changes for the 2008 competition (2008), http://megatron.iiia.csic.es/art-testbed/changes_2008.htm 